\title{
Ponderomotive entanglement purification
}

\author{
Stefano Mancini \\ INFM, Dipartimento di Fisica, Università di Milano, Via Celoria 16, I-20133 Milano, Italy
}

(November 21, 2018)

\begin{abstract}
It is shown that ponderomotive force can be used to purify entangled states. The protocol is based on the possibility to exploit such force for a local quantum nondemolition measurement of the total excitation number of continuos variable entangled pairs.

PACS number(s): 03.65.Bz, 42.50.Vk, 03.67.Hk
\end{abstract}

\section{INTRODUCTION}

Entanglement [1] has been recognized as one of the most puzzling features of Quantum Mechanics, being also the basis of quantum information processing [2]. However, to realize protocols like quantum key distribution or quantum teleportation [3] one needs of maximally entangled states which are not easy to generate (due to loss and decoherence). Thus entanglement purification is often required to distill maximum entangled states from several pairs of partial entangled states by using local quantum operation and classical communications 任.

For qubit systems, efficient entanglement purification protocols have been found [4. Recently, quantum information protocols have been also extended to continuos variable systems [5]. In particular, in Ref. [6] an efficient continuos variable entanglement purification protocol has been proposed. Here, a physical implementation of this protocol, based on ponderomotive effect is studied. The protocol is based on the possibility of a local quantum nondemolition (QND) measurement of the total excitation number of continuos variables. On the other hand the principle underlying a method of measuring electromagnetic energy avoiding photon absorption has been known for approximately a hundred years: measure the electromagnetic pressure associated with the energy by an experiment like that of Peotr N. Lebedev, and from that pressure infer the energy. Hence, we shall consider such ponderomotive meter [7] for entanglement purification.

\section{THE MODEL}

It is well known [8] that two-mode squeezed states can be generated as intracavity stationary state from the broadband squeezed light provided by a Nondegenerate Optical Parametric Amplifier (NOPA) operating below threshold. Let us now suppose that the two modes characterize two different cavities. The latter are also placed in two distant sides, say $A$ and $B$, respectively. Then, the steady state of the cavity modes $a_{1}$ (in the A side) and $b_{1}$ (in the $\mathrm{B}$ side) can be written as $S(r)|0\rangle_{a_{1}}|0\rangle_{b_{1}}$ where $S(r)=\exp \left[r\left(a_{1}^{\dagger} b_{1}^{\dagger}-a_{1} b_{1}\right)\right]$ is the squeezing operator with the squeezing parameter $r$ [9]. Now, let us consider another pair of modes, say $a_{2}$ (in the A side) and $b_{2}$ (in the $\mathrm{B}$ side), in the same state, i.e. $S(r)|0\rangle_{a_{2}}|0\rangle_{b_{2}}$. Furthermore, suppose that the $a_{i}\left(b_{i}\right)$ modes $i=1,2$, belong to the same cavity in the A (B) side. For instance, they have the same frequency $\omega_{a}\left(\omega_{b}\right)$, but they distinguish for their polarization. Then, the state of the whole system is $|\psi\rangle_{1} \otimes|\psi\rangle_{2}$, where $|\psi\rangle_{i}(i=1,2)$ is a two-mode squeezed state

$$
|\psi\rangle_{i}=\sqrt{1-\lambda^{2}} \sum_{n=0}^{\infty} \lambda^{n}|n\rangle_{a_{i}} \otimes|n\rangle_{b_{i}}
$$

with $\lambda=\tanh r$. The state $|\psi\rangle_{1} \otimes|\psi\rangle_{2}$ represents two pairs of partial entangled states.

Following the avenue sketched in Ref. [6] we choose to make a QND measurement of the total number of photons in the A side. To this end we exploit a ponderomotive meter [7]. A suspended mirror provides an intuitive realization of such meter [10], however, a more realistic apparatus can be realized by using for example a cavity made by a piezoelectric crystal coated on both sides [11]. For a piezoelectric crystal, the length variation due to radiation pressure can be measured by a suitable electric circuit.

A simple model to describe the coupling of a ponderomotive meter with the A modes, is obtained as straightforward generalization of that presented in Refs. 12,13]. Let $c$ the oscillatory mode characterizing the meter and $\Omega$ its frequency, then the Hamiltonian (in natural unit) governing the measurement dynamics is 


$$
H=\omega_{a} \sum_{i=1}^{2} a_{i}^{\dagger} a_{i}+\omega_{b} \sum_{i=1}^{2} b_{i}^{\dagger} b_{i}+\Omega c^{\dagger} c-g\left[\sum_{i=1}^{2} a_{i}^{\dagger} a_{i}\right]\left(c+c^{\dagger}\right),
$$

with $g$ the coupling constant between signal and meter. The interaction part of the Hamiltonian (fourth term in Eq.(22)), simply represents the effect of the radiation pressure force which causes the instantaneous displacement $x$ of the meter [14]. Here, $x=\left(c+c^{\dagger}\right) / \sqrt{2}, p=-i\left(c-c^{\dagger}\right) / \sqrt{2}$ are the meter position and momentum quadratures respectively.

On the other hand, the meter would be connected with a readout apparatus, and this can be modeled as a heath bath [15,16. In particular, a meter undergoing quantum Brownian motion can be described by the following master equation [8]

$$
\dot{\rho}=-i[H, \rho]-i \frac{\gamma}{2}[x,\{p, \rho\}]-\frac{\gamma}{\beta}[x,[x, \rho]],
$$

where $\gamma$ is the damping rate and $\beta$ the parameter proportional to the inverse of the temperature. By assuming the meter initially in the vacuum, the initial state of the whole system is

$$
\rho_{0}=|\psi\rangle_{1}\langle\psi|\otimes| \psi\rangle_{2}\langle\psi|\otimes| 0\rangle_{m}\langle 0| .
$$

Since, from Eq.(2), $H$ commutes with the total number operator in the A side, $N=\sum_{i=1}^{2} a_{i}^{\dagger} a_{i}$, one can solve Eq.(3) by introducing the following characteristic function

$$
\chi_{N, M}\left(\mu, \mu^{*}, t\right)=\operatorname{Tr}_{m}\left\{\rho_{N, M}(t) e^{\mu c^{\dagger}} e^{-\mu^{*} c}\right\}
$$

where $\rho_{N, M}$ indicate the density matrix elements of radiation fields built up with 'ket' and 'bra' having total number of photons $N$ and $M$ respectively. Then, Eq.(3) can be transformed in a partial differential equation for the characteristic function (5). The latter, in turn, due to its linearity allows a simple Gaussian solution. Without going down in the details of calculations, it is possible to see that, in the limit $t \gg \gamma^{-1}$, the following steady state is reached

$$
\begin{aligned}
\rho_{s s}=\sum_{N=0}^{\infty} \sum_{n_{1}=0}^{N} \sum_{m_{1}=0}^{N}\left(1-\lambda^{2}\right)^{2} \lambda^{2 N} & \left|n_{1}\right\rangle_{a_{1}}\left\langle m_{1}|\otimes| N-n_{1}\right\rangle_{a_{2}}\left\langle N-m_{1}\right| \\
& \otimes\left|n_{1}\right\rangle_{b_{1}}\left\langle m_{1}|\otimes| N-n_{1}\right\rangle_{b_{2}}\left\langle N-m_{1}\right| \\
& \otimes\left[D(-\kappa N) \rho_{t h}^{(m)} D^{\dagger}(-\kappa N)\right],
\end{aligned}
$$

where $D$ denotes the displacement operator [17, and $\kappa=g / \Omega$. Moreover,

$$
\rho_{t h}^{(m)}=\left(1-e^{-\beta}\right) \sum_{n=0}^{\infty} e^{-n \beta}|n\rangle_{m}\langle n|,
$$

is the thermal state of the meter. Practically, the dynamics leading to the state (6) corresponds to the 'collapse' of wavefunction due to the measurement process [18]. Once $\gamma$ becomes large (strong meter-environment interaction), the model provides an idealized limit of real measurement process. Notice that by using the quantum Brownian motion master equation no Rotating Wave Approximation has been invoked, differently from Ref. [16] where the quantum optical master equation was used in an analog measurement model.

The radiation fields state corresponding to the meter outcome $x$ is then obtained by projecting (6) onto the meter eigenstate $|x\rangle_{m}$ [18, that is

$$
\begin{aligned}
\rho^{(r)}= & \mathcal{N} \sum_{\substack{N=0 \\
n_{1}}}^{\infty} \sum_{n_{1}=0}^{N} \sum_{m_{1}=0}^{N}\left(1-\lambda^{2}\right)^{2} \lambda^{2 N}{ }_{m}\left\langle x\left|\left[D(-\kappa N) \rho_{t h}^{(m)} D^{\dagger}(-\kappa N)\right]\right| x\right\rangle_{m} \\
& \otimes\left|N-n_{1}\right\rangle_{a_{2}}\left\langle N-m_{1}|\otimes| n_{1}\right\rangle_{b_{1}}\left\langle m_{1}|\otimes| N-n_{1}\right\rangle_{b_{2}}\left\langle N-m_{1}\right|,
\end{aligned}
$$

where the normalization constant is given by

$$
\mathcal{N}^{-1}=\sum_{N=0}^{\infty}{ }_{m}\left\langle x\left|\left[D(-\kappa N) \rho_{t h}^{(m)} D^{\dagger}(-\kappa N)\right]\right| x\right\rangle_{m},
$$


and

$$
{ }_{m}\left\langle x\left|\left[D(-\kappa N) \rho_{t h}^{(m)} D^{\dagger}(-\kappa N)\right]\right| x\right\rangle_{m}=\frac{1-e^{-\beta}}{\sqrt{\pi}} \sum_{n=0}^{\infty} \frac{e^{-\beta}}{2^{n} n !} \exp \left[-(x+\sqrt{2} \kappa N)^{2}\right] H_{n}^{2}[x+\sqrt{2} \kappa N],
$$

with $H_{n}($.$) the Hermite polynomials.$

The normalization constant (9) depends on the value of the outcome $x$, and gives the meter position distribution $P(x)$, that is $P(x) \equiv \mathcal{N}^{-1}$. Let us now assume for the sake of simplicity $\beta=\infty$, i.e. the meter goes in a coherent state of amplitude $-\kappa N$ 19]. Then, Fig.(1) shows a typical distribution of outcomes. Different peaks correspond to different values of $N$, from the right to the left $N=0,1 \ldots$. Notice that in order to distinguish all possible values of $N$ one should have the minimum amount of displacement (corresponding to $\kappa$ ) greater than the width of a coherent state, i.e. $1 / 2$. Therefore, the measurement efficiency increases monotonically with the signal-meter correlation.

\section{ENTANGLEMENT QUANTIFICATION}

At the end of the measurement process the partial density operator can be obtained by tracing Eq.(8) over the A side

$$
\begin{aligned}
\rho_{B}^{(r)}(x) & =\operatorname{Tr}_{A}\left\{\rho^{(r)}(x)\right\} \\
& =\mathcal{N}\left(1-\lambda^{2}\right)^{2} \sum_{N=0}^{\infty} \sum_{n_{1}=0}^{N} \lambda^{2 N}{ }_{m}\left\langle x\left|\left[D(-\kappa N) \rho_{t h}^{(m)} D^{\dagger}(-\kappa N)\right]\right| x\right\rangle_{m}\left|n_{1}\right\rangle_{b_{1}}\left\langle n_{1}|\otimes| N-n_{1}\right\rangle_{b_{2}}\left\langle N-n_{1}\right| .
\end{aligned}
$$

One can easily see from the above expression (11) that the state $\left|n_{1}\right\rangle_{b_{1}}\left|n_{2}\right\rangle_{b_{2}}$ is an eigenstate of the reduced density operator

$$
\rho_{B}^{(r)}(x)\left(\left|n_{1}\right\rangle_{b_{1}}\left|n_{2}\right\rangle_{b_{2}}\right)=\Lambda_{n_{1}, n_{2}}(x)\left(\left|n_{1}\right\rangle_{b_{1}}\left|n_{2}\right\rangle_{b_{2}}\right)
$$

whose eigenvalues are

$$
\Lambda_{n_{1}, n_{2}}(x)=\mathcal{N}\left(1-\lambda^{2}\right)^{2} \lambda^{2\left(n_{1}+n_{2}\right)}{ }_{m}\left\langle x\left|\left[D(-\kappa N) \rho_{t h}^{(m)} D^{\dagger}(-\kappa N)\right]\right| x\right\rangle_{m} .
$$

Now, the measure of entanglement for a pure bipartite system is just the Von Neumann entropy of either partial density operator [20]. Hence, it can be written as [21]

$$
\mathcal{E}(x)=-\sum_{n_{1}, n_{2}=0}^{\infty} \Lambda_{n_{1}, n_{2}}(x)\left[\log _{2} \Lambda_{n_{1}, n_{2}}(x)\right],
$$

where the dependence from $x$ indicates the dependence from the outcome of measurement.

Furthermore, the quantity

$$
\Gamma(x)=\frac{\mathcal{E}(x)}{\mathcal{E}_{0}},
$$

defines the entanglement increase ratio, once $\mathcal{E}_{0}$ identifies the initial amount of entanglement. Of course, if $\Gamma(x)>1$, the state after the measurement is more entangled with respect to the initial one. Prior the measurement we have $\Lambda_{n_{1}, n_{2}}=\left(1-\lambda^{2}\right)^{2} \lambda^{2\left(n_{1}+n_{2}\right)}$ and therefore

$$
\mathcal{E}_{0}=2\left[\cosh ^{2} r \log _{2}\left(\cosh ^{2} r\right)-\sinh ^{2} r \log _{2}\left(\sinh ^{2} r\right)\right],
$$

which is obviously not depending on $x$. Furthermore, it results equal to the entanglement of a single pair times the number of pairs.

In Fig. (2) the entanglement increasing ratio $\Gamma$ is shown as function of the measured value of position quadrature $x$ and coupling constant $\kappa$. We may see that for $\kappa=0$ the entanglement remain the same since the meter is practically uncorrelated with the signal. On the other hand, the result $x=0$ (corresponding to $N=0$ ) never increases the entanglement. For $\kappa \neq 0, \Gamma$ grows up approximately as $x$ diminishes; however, one should take into account that the 
probability to get large negative values of $x$ decreases, as can be seen from Fig.(1). Then, it is useful to introduce the probability of success of the protocol

$$
P_{S}=\int_{\Gamma>\epsilon} d x P(x),
$$

where integration is performed over the set $\{x \in \mathbf{R} \mid \Gamma(x)>\epsilon\}$, with $\epsilon$ defining the lower limit for the success $(\epsilon \geq 1)$. The quantity $P_{S}$ is plotted in Fig.(33.a) for $\epsilon=1$ (solid line) and for $\epsilon=1.5$ (dashed line). One can see that an higher increment of the degree of entanglement is achieved by increasing the value of $\kappa$, but with a lower probability.

It is also instructive to consider the efficiency of the protocol. It can be defined as

$$
\Xi=1-\frac{1}{\Upsilon},
$$

where

$$
\Upsilon=\frac{\int_{\Gamma>\epsilon} d x \Gamma(x) P(x)}{\int_{\Gamma>\epsilon} d x P(x)},
$$

represents the average of the entanglement increase ratio weighted on the probability of success. It always results $\Upsilon \geq 1$ and $\Xi \leq 1$. Fig.(3.b) (solid line) shows the efficiency of the protocol. Contrarily to what one can expect for a better resolution of the number of photons, the efficiency does not increase monotonically with $\kappa$. Instead, there exist a particular value of $\kappa$ that maximizes the efficiency, and it is slightly greater than the width of the final meter state. In fact, for such value of $\kappa$, a measurement giving a result, say $x$, different from zero cannot be identified with a particular value of $N$, but it practically corresponds to any $N \neq 0$, thus improving the efficiency of the purification process. As matter of fact we have seen in Fig.(2) that $\Gamma$ exceeds 1 for $N \neq 0$ (essentially $x<0$ ). However, this limit, hence the optimal value of $\kappa$, also depends on the initial amount of entanglement, i.e. on the squeezing parameter $r$.

Fig.(3.b) (dashed line) shows the thermal effects on the efficiency of the purification protocol. In such a case the distribution $P$ becomes broader, hence, the protocol results less efficient for $\kappa$ maximizing $\Xi$ at $\beta=\infty$. However, the effect of temperature is somehow equivalent to decreasing the coupling constant $\kappa$, in fact for $\kappa \rightarrow 0$ it should be $\Gamma(x) \rightarrow 1$. Hence the thermal effect can be hindered by increasing $\kappa$. The extension of previous arguments yields $\kappa$ slightly greater than $\beta^{-1}$ as criteria for efficient protocol. Notice that smaller values of $\beta$ could be considered obtaining the same behavior [19].

Finally, the developed theory can be extended to consider more than two radiation field pairs per side. Consequently, the efficiency of the protocol can be improved. Furthermore, in this scheme, all the modes in one side can be considered belonging to the same cavity, at least in the side where the measurement is performed (A). However, in that case the coupling constant becomes mode depending since we have to account for the mode spacing.

\section{CONCLUSIONS}

In conclusion, the role of a ponderomotive meter in entanglement purification process is studied. It is found that there exist optimal values of the signal-meter coupling constant, contrarily from what one can expect by a simple analysis on indirect measurement. The purification process results sensible to the thermal effects introduced by the meter, however, appropriate signal-meter coupling reduces such detrimental effects. It is worth noting that a wide range of $\kappa$ values are accessible for movable mirrors with the present technology [22]. Otherwise, microfabricated cantilevers 223 could be also employed. Furthermore, a large number of pairs involved in the process renders it more efficient.

Since radiation pressure allows to couple macroscopic objects (like mirrors) with microscopic systems (like cavity modes), it provides a unique bridge between quantum and classical worlds. Hence, optomechanical systems are worth studying, also for quantum information processing.

Finally, ponderomotive effects come out also in the dispersive regime of atom-field interaction [24], where the atomic momentum becomes sensible to the photon statistics, and a scheme analogous to that described can be developed with atoms crossing a cavity. In such a case the thermal effects should be less significant. 


\section{ACKNOWLEDGEMENTS}

The author gratefully acknowledge financial support from Università di Camerino, Italy, under the Project 'Giovani Ricercatori'.

[1] E. Schrödinger, Naturwiss. 23, 807 (1935).

[2] C. H. Bennett and D. P. DiVincenzo, Nature 404, 247 (2000).

[3] C. H. Bennett, Phys. Today, 48(10), 24 (1995).

[4] C. H. Bennett, G. Brassard, S. Popescu, B. Schumacher, J. A. Smolin and W. K. Wootters, Phys. Rev. Lett. 76, 722 (1996); C. H. Bennet, H. J. Herbert, S. Popescu and B. Schumacher, Phys. Rev. A 53, 2046 (1996); C. H. Bennett, D. P. DiVincenzo, J. A. Smolin and W. K. Wootters, Phys. Rev. A 54, 3824 (1996).

[5] L. Vaidman, Phys. Rev. A 49, 1473 (1994); S. L. Braunstein and J. Kimble, Phys. Rev. Lett. 80, 869 (1998).

[6] Lu-Ming Duan, G. Giedke, J. I. Cirac and P. Zoller, Phys. Rev. Lett. 84, 40002 (2000); Phys. Rev. A 62, 032304 (2000).

[7] V. B. Braginsky and F. Ya. Khalili, Quantum Measurement, (Cambridge Univ. Press, Cambridge, 1992).

[8] C. W. Gardiner, Quantum Noise, (Springer, Berlin, 1991); C. W. Gardiner and P. Zoller, Quantum Noise, (Springer, Berlin, 1999).

[9] B. L. Schumacher and C. M. Caves, Phys. Rev. A 31, 3093 (1985).

[10] K. Jacobs, P. Tombesi, M. J. Collett and D. F. Walls, Phys. Rev. A 49, 1961 (1994).

[11] M. Pinard, C. Fabre and A. Heidmann, Phys. Rev. A 51, 2443 (1995).

[12] S. Mancini, V. I. Man'ko and P. Tombesi, Phys. Rev. A 55, 3042 (1997).

[13] S. Bose, K. Jacobs and P. Knight, Phys. Rev. A 56, 4175 (1997).

[14] A. F. Pace, M. J. Collett and D. F. Walls, Phys. Rev. A 47, 3173 (1993); S. Mancini and P. Tombesi Phys. Rev. A 49, 4055 (1994).

[15] W. H. Zurek, Phys. Rev. D 24, 1516 (1981); W. H. Zurek, Phys. Rev. D 26, 1862 (1982).

[16] D. F. Walls, M. J. Collett and G. J. Milburn, Phys. Rev. D 32, 3208 (1985).

[17] R. J. Glauber, Phys. Rev. 131, 2766 (1963).

[18] J. von Neumann, Mathematical Foundations of Quantum Mechanics, (Princeton Univ. Press, 1955).

[19] To be rigorous, the master equation (3) is only valid for $\beta \ll 1$, however, the opposite limit has been chosen to easily describe the qualitative behavior of the system.

[20] C. H. Bennet, H. J. Herbert, S. Popescu and B. Schumacher, Phys. Rev. A 53, 2046 (1996); S. Popescu and D. Rohrlich, Phys. Rev. A 56, R3319 (1997).

[21] S. Parker, S. Bose and M. B. Plenio, Phys. Rev. A 61, 032305 (2000).

[22] I. Tittonen, T. Kalkbrenner, G. Breitenbach, T. Muller, R. Conradt, S. Schiller, E. Steinslad, N. Blanc and N. F. de Rooij, Phys. Rev. A 59, 1038 (1999); Y. Hadjar, P. Cohadon, C. G. Aminoff, M. Pinard and A. Heidmann, Europhys. Lett. 46, 545 (1999); P. F. Cohadon, A. Heidmann and M. Pinard, Phys. Rev. Lett. 83, 3174 (1999).

[23] A. N. Cleland and M. L. Roukes, Appl. Phys. Lett. 69, 2653 (1996).

[24] D. F. Walls and G. J. Milburn, Quantum Optics, (Springer, Berlin, 1995); G. Nogues, A. Rauchenbeutel, S. Osnaghi, M. Brune, J. M. Raimond and S. Haroche, Nature 400, 239 (1999). 


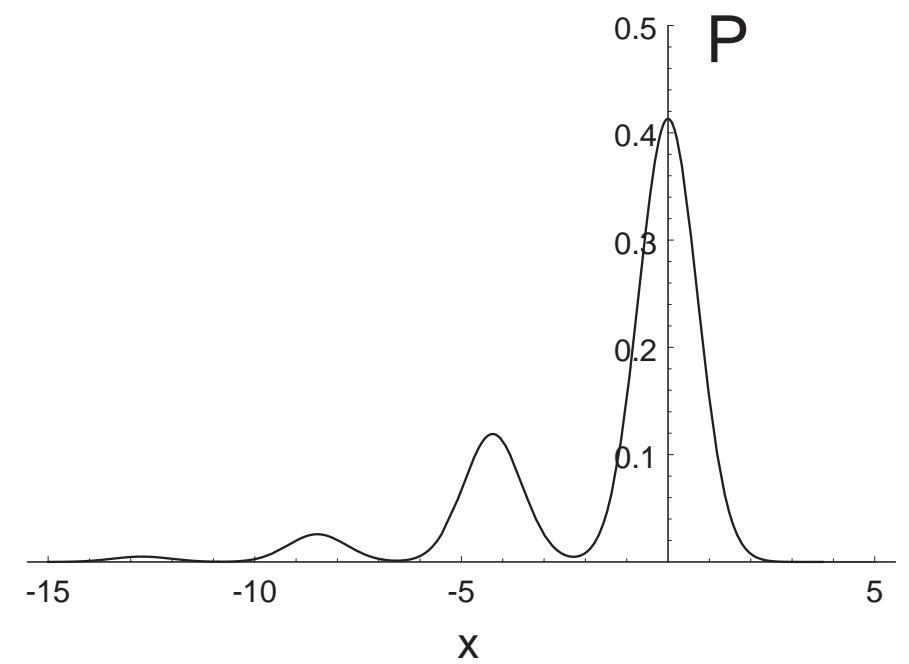

FIG. 1. Meter position probability distribution $P(x)$. Here $\kappa=3, \beta=\infty$ and $r=0.4$.

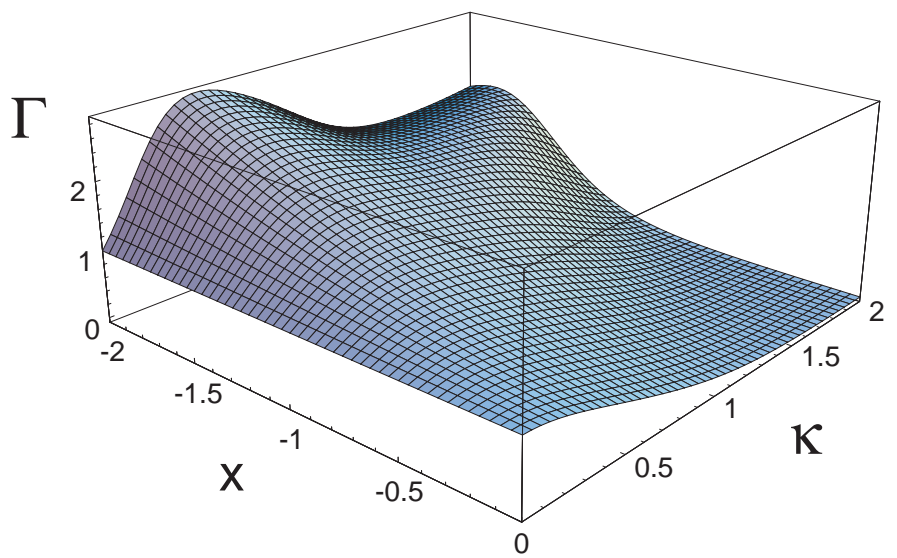

FIG. 2. The entanglement purification ratio $\Gamma$ as function of position quadrature $x$ and coupling constant $\kappa$. The parameters are $\beta=\infty$ and $r=0.3$. 
a)
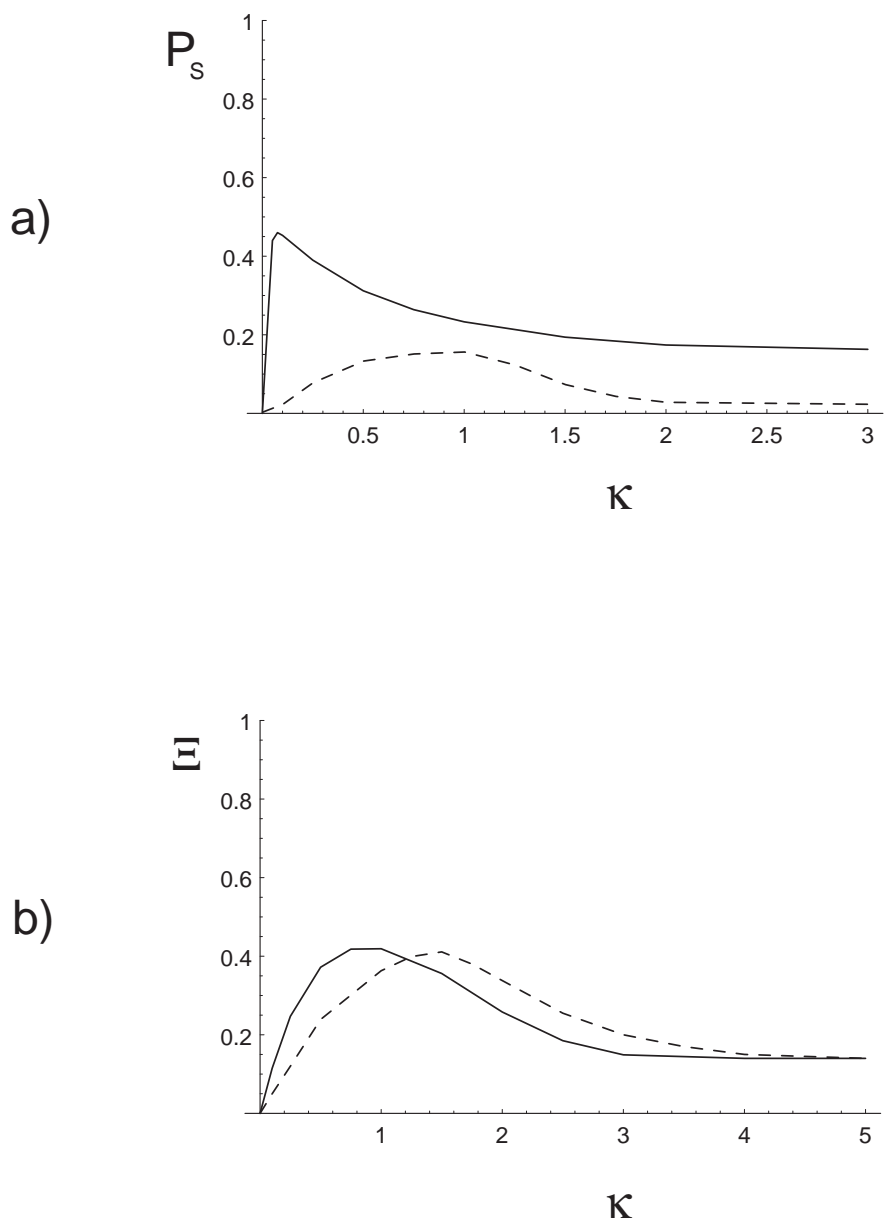

FIG. 3. The success probability $P_{S}$ is shown in figure a) vs the coupling constant $\kappa$. The solid line is for $\epsilon=1$ and the dashed line for $\epsilon=1.5$. The efficiency $\Xi$ of the purification protocol is shown in figure b) vs the coupling constant $\kappa$ for $\epsilon=1$. The solid line is for $\beta=\infty$ and the dashed line for $\beta=1$. In both figures a) and b) $r=0.3$. 